\title{
Spatio-temporal Talbot phenomenon using metamaterial composite right/left-handed leaky-wave antennas
}

\author{
J. S. Gómez-Díaz, ${ }^{1, a)}$ A. Alvarez-Melcon, ${ }^{1}$ S. Gupta, ${ }^{2}$ and C. Caloz ${ }^{2}$ \\ ${ }^{1}$ Technical University of Cartagena, Campus Muralla del Mar-Antiguones, Cartagena, Murcia, 30202 Spain \\ ${ }^{2}$ École Polytechnique de Montréal, 2500, ch. de Polytechnique Montreal, Quebec, H3T 1J4, Canada
}

(Received 27 May 2008; accepted 22 September 2008; published online 17 November 2008)

\begin{abstract}
A spatial-temporal Talbot phenomenon, based on metamaterial composite right/left-handed (CRLH) leaky-wave antennas (LWAs), is presented. This phenomenon, reported in the microwave domain, is based on the combination of the conventional spatial monochromatic Talbot effect and the transient (polychromatic) character of the pulse radiation phenomenon in the LWA structure. When the elements of a periodic CRLH LWA array are fed simultaneously by an input pulse, the spatial beams corresponding to different temporal frequencies constructively interfere in space so as to form a self-imaged pattern constituted by narrow Talbot zones. This Talbot effect is spatial-temporal since the Talbot zones are localized both in space and time. The phenomenon is analyzed theoretically and validated numerically for the case of narrow-band pulses. (c) 2008 American Institute of Physics. [DOI: 10.1063/1.3013905]
\end{abstract}

\section{INTRODUCTION}

The Talbot effect was discovered by Talbot ${ }^{1}$ in 1836 and has been widely applied in different areas of research. ${ }^{2-4}$ It is a constructive interference self-imaging effect occurring at specific distances (Talbot planes) due to diffraction from a periodic spatial object (for instance, a diffraction grating) under monochromatic ${ }^{5}$ or polychromatic ${ }^{6}$ illumination. The equations that describe this diffraction phenomenon, under paraxial (Fresnel) approximation, are identical to those that describe the temporal propagation (under first-order approximation) of pulses in a dispersive dielectric waveguide, leading to the temporal counterpart of the Talbot effect. ${ }^{7}$ This phenomenon occurs when a signal, which is periodic in time, propagates along a first-order dispersive medium (such as an optical fiber). In this case the input pulse train is replicated at the Talbot distance.

The advent of electromagnetic metamaterials, such as composite right/left-handed transmission lines (CRLH TL) metamaterials, has paved the road for novel dispersive effects and devices in the microwave domain. ${ }^{8}$ Due to their inherent broadband nature, CRLH TL metamaterials can be used in the impulse regime. Initial reports in this field include a tunable pulse delay line, ${ }^{9}$ a temporal Talbot effect transmission line pulse multiplier, ${ }^{10}$ and a real-time spectrogram analyzer based on CRLH leaky-wave antennas (LWAs). ${ }^{11}$

In this contribution, a spatial-temporal Talbot phenomenon is introduced. This phenomenon, reported in the microwave domain, is based on the combination of the conventional monochromatic spatial Talbot effect and the transient (polychromatic) effect of pulse radiation by a LWA. To produce this phenomenon, an array of CRLH LWAs is fed simultaneously at all of its elements by a modulated pulse with center frequency located at the transition frequency of the LWAs. ${ }^{12}$ The beams radiated by the different elements gen-

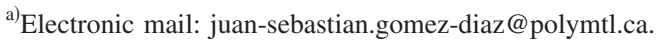

erate an interference pattern that self-image the spatial pulse distribution along the antennas at the Talbot distance. Furthermore, an increase in the repetition rate of this spatial distribution occurs at the fractional Talbot distances. The CRLH LWA, which is sufficiently directive for a given pulse bandwidth, generates a paraxial diffraction (i.e., radiation) of the beams, leading to the spatial Talbot effect. This, combined with the transient nature of the pulsed antenna radiation, leads to the spatial-temporal Talbot phenomenon. In addition, the self-imaging effect replicates the spatial variation of the pulses as a function of time at each Talbot zones due to the pulses propagation along the CRLH LWAs.

The paper is organized as follows. Section II briefly reviews the conventional spatial and temporal Talbot effects and presents the fundamentals of the CRLH spatial-temporal Talbot phenomenon. Section III derives the mathematical calculation for the integer and fractional Talbot distances. Section IV demonstrates and characterizes the spatialtemporal Talbot phenomenon using an exact time-domain Green's function approach. Finally, conclusions are given in Sec. V.

\section{PRINCIPLE}

This section briefly reviews the spatial and temporal Talbot effects, which are based on spatial dispersion and temporal frequency dispersion, respectively. The spatialtemporal Talbot phenomenon, based on the impulse-regime properties of the CRLH LWA, is presented and discussed.

\section{A. Spatial and temporal Talbot phenomena}

The spatial Talbot phenomenon is observed when a monochromatic wave is transmitted through (or reflected from) a periodically distributed spatial object. An exact image of the original object appears at a specific distance called the integer Talbot distance and denoted by $z_{T}$. Also, additional images, with periods that are multiple of the original 
object period, appear at fractional distances of $z_{T}$, i.e., at $z_{T} / m(m \in \mathbb{N})$. In general, the integer/fractional Talbot distances are given by

$$
d_{s}=\frac{s}{m} \frac{X^{2}}{\lambda}
$$

where $X$ is the object spatial period, $\lambda$ is the wavelength, $s$, $m \in \mathbb{N}$, and $(s / m)$ is an integer number (for $z_{T}$ and its multiples) or an irreducible rational number (for the fractional Talbot distances). The spatial Talbot effect has been used in various applications, such as array illumination, ${ }^{2}$ cavity and phase locking for laser arrays, ${ }^{3}$ and holographic multiplexing storage. $^{4}$

The temporal counterpart of the spatial Talbot phenomenon occurs when a periodic temporal signal propagates through a dispersive medium with first-order dispersion. ${ }^{7}$ This may be shown from the mathematical equivalence of paraxial Fresnel diffraction in space and pulse propagation in a first-order dispersive medium. ${ }^{7}$ In case of the temporal Talbot effect, the input pulse train is exactly replicated along the medium at the Talbot distance, while a multiplication of the repetition rate of the periodic signal is obtained at fractional Talbot distances. The integer/fractional Talbot distances are given by

$$
d_{t}=\frac{s}{m} \frac{T_{r}^{2}}{2 \pi\left|\beta_{2}\right|},
$$

where $T_{r}$ is the temporal repetition period, $\beta_{2}$ is the group velocity dispersion parameter of the medium $s, m \in \mathbb{N}$, and $(\mathrm{s} / \mathrm{m})$ is an integer number (for $z_{T}$ and its multiples) or an irreducible rational number (for the fractional Talbot distances). In practical applications, such as multiplication of the repetition rate of a periodic pulse $\operatorname{train}^{13}$ or pulse compression, ${ }^{14}$ optical fibers or chirped fiber gratings may be used. The temporal Talbot phenomenon was also theoretically demonstrated at microwave frequencies along a dispersive CRLH transmission line. ${ }^{10}$

\section{B. Composite right/left-handed leaky-wave antennas}

Recently, the introduction of novel metamaterial structures, and in particular CRLH TL metamaterials, has led to novel and efficient LWAs. ${ }^{8}$ These antennas provide fullspace scanning capability, from backfire $\left(\theta=-90^{\circ}\right)$ to endfire $\left(\theta=90^{\circ}\right)$, including broadside $\left(\theta=0^{\circ}\right)$. The main beam angle $(\theta)$ measured from the normal to the antenna is given by the scanning law

$$
\sin \theta=\frac{\beta(\omega)}{k_{0}},
$$

where $k_{0}$ is the free-space wave number and $\beta(\omega)$ is the propagation constant along (or dispersion relation of) the CRLH structure, which in the infinitesimal limit is given by

$$
\beta(\omega)=\frac{\omega}{\omega_{R}^{\prime}}-\frac{\omega_{L}^{\prime}}{\omega},
$$

with

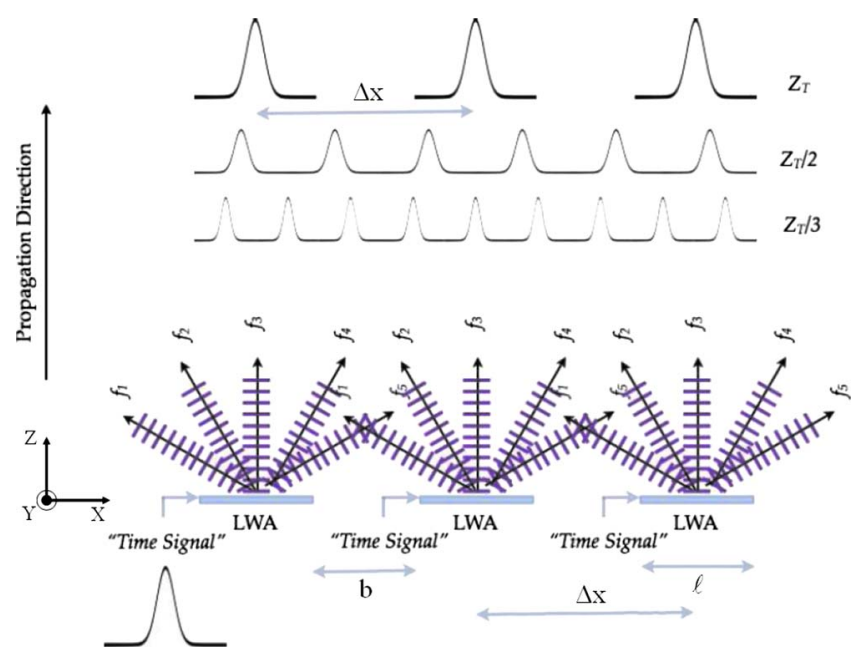

FIG. 1. (Color online) Proposed CRLH LWA array configuration for the investigation of the spatial-temporal Talbot effect. Each antenna radiates the different frequency components of the input modulated pulse to different angles of space. For the sake of simplicity, only the envelopes of the pulses at one Talbot plane are shown.

$$
\omega_{R}^{\prime}=\frac{1}{\sqrt{L_{R}^{\prime} C_{R}^{\prime}}} \quad \text { and } \quad \omega_{L}^{\prime}=\frac{1}{\sqrt{L_{L}^{\prime} C_{L}^{\prime}}},
$$

where $L_{R}^{\prime}, C_{R}^{\prime}$ and $L_{L}^{\prime}, C_{L}^{\prime}$ are the per-unit-length and timesunit-length circuit parameters. ${ }^{8}$ They define the right-handed and left-handed contributions of the CRLH structure. Defining the transition frequency as $\omega_{0}=\sqrt{\omega_{R}^{\prime} \omega_{L}^{\prime}}$, we have $\beta(\omega$ $\left.<\omega_{0}\right)<0$, which corresponds to the left-handed (or backward-wave or negative refractive index) band, and provides negative radiation angles according to Eq. (3). Moreover, $\beta\left(\omega>\omega_{0}\right)>0$, which corresponds to the right-handed (or forward-wave or positive refractive index) band, and provides positive radiation angles.

The scanning law of Eq. (3) reveals that each frequency is mapped onto a specific radiation angle in space. When the CRLH LWA is operated in the impulse regime, the different spectral components of the broadband pulse at the input of the LWA are separated in space following this scanning law. Thus, a LWA performs a spatial-spectral decomposition ${ }^{11}$ of the input signal. This may be interpreted as spatial dispersion, where the different frequencies are separated in space due to frequency-dependent radiation angles.

\section{Spatio-temporal Talbot phenomenon}

The spatial-temporal Talbot phenomenon occurs when the CRLH LWA array elements (or possibly diffraction grating elements) are simultaneously fed with a (single) temporal pulse. Due to the spatial-spectral decomposition property of the antennas, the pulses are spectrally decomposed in space following the CRLH beam-scanning law of Eq. (3), as illustrated in Fig. 1. The spatial beams corresponding to each temporal frequency interfere in space creating an interference pattern (i.e., the spatial Talbot effect). Due to the nonzero bandwidth of the input pulse, the Talbot planes occurring in the case of the monochromatic wave are replaced by Talbot zones $z_{T} \pm \Delta z_{T} / 2$ corresponding to the bandwidth 
$\omega_{0} \pm \Delta \omega_{0} / 2$ of the pulse. This Talbot effect is spatial temporal since the Talbot zones are localized both in space and time.

In the case of narrow-band pulses, the Talbot zones may be localized at their center $z_{T}$ related to the transition frequency $\omega_{0}$, corresponding to broadside $\left(\theta=0^{\circ}\right)$ radiation, of the CRLH LWA. Furthermore, this interference at the Talbot plane reproduces the spatial distribution of the pulses along the CRLH lines, which are varying as a function of time, due to their propagation. Thereby, instead of the regular space or time domain, this phenomenon is observed in the combined spatial-temporal domain, and the spatial images are reconstructed at a specific position $\left(z_{T}\right)$ as a function of time.

\section{DERIVATION OF THE TALBOT DISTANCE}

This section presents the detailed mathematical analysis of the spatial-temporal Talbot phenomenon, leading to a close-form expression for the Talbot distance. Consider an infinite array of CRLH LWAs, with antenna element spacing $b$ and element length $\ell$. All of the elements are fed simultaneously with the same input pulse, as illustrated in Fig. 1. This pulse is modulated at the CRLH transition frequency $\omega_{0}$ and, assuming the phasor time dependence $e^{+j \omega t}$, it may be expressed as

$$
\Psi(t)=\Psi_{0}(t) e^{j \omega_{0} t},
$$

where $\Psi_{0}(t)$ is a slowly varying envelope and $\omega_{0}$ is the modulation frequency.

Since the elements of the array are all excited simultaneously by the same pulse and are all identical, the field distribution along the overall array is necessarily periodic with the spatial period $\Delta x$, which is independent of time. Consequently, the Talbot distance $z_{T}$ will also be independent of time. Rather, as the signals propagate along the CRLH structures, the Talbot image will experience a continuous shift along the $x$-axis following this propagation.

Due to this time-independence of the Talbot distance, the spatial distribution of the field along each CRLH array element, denoted by $A_{e}(x, z=0)$, is considered at the fixed time $t=t^{\prime}$, which may be seen as a "snapshot" of the pulse along the element. Taking all the elements of the array into account, the spatial distribution of the field along the overall array takes the periodic form

$$
A_{a}(x, z=0)=\sum_{p=-\infty}^{+\infty} A_{e}(x, z=0) * \delta(x-p \Delta x),
$$

where the symbol "*” represents the convolution operation. Taking the Fourier transform of this expression yields

$$
\tilde{A}_{a}\left(k_{x}, z=0\right)=\Delta k_{x} \sum_{p=-\infty}^{+\infty} \tilde{A}_{e}\left(k_{x}=p \Delta k_{x}\right) \delta\left(k_{x}-p \Delta k_{x}\right)
$$

where $\Delta k_{x}=2 \pi / \Delta x$, with $\Delta x=b+\ell$, is the repetition frequency.

On the other hand, the transfer function of the CRLH LWA, assuming plane-wave propagation, ${ }^{15}$ is given by ${ }^{16}$

$$
\tilde{H}\left(k_{x}, z\right)=e^{-j k_{z} z}=e^{-j \sqrt{k_{0}^{2}-k_{x}^{2}} z}=e^{-j k_{0} \sqrt{1-k_{x}^{2} / k_{0}^{2}} z},
$$

where the Helmoltz equation implies that $k_{0}^{2}=k_{z}^{2}+k_{x}^{2}$. This relation may be simplified as follows. First the CRLH LWA scanning law [Eq. (3)] implies $\beta / k_{0}=k_{x} / k_{0}=\sin \theta$. Here we assumed that the propagation constant along the CRLH in Fig. 1 is $\beta=k_{x}$. Second, the modulation frequency has been chosen as the transition frequency $\omega_{0}$, corresponding to $\theta\left(\omega_{0}\right)=0^{\circ}$, so that $k_{x} / k_{0}\left(\omega_{0}\right)=0^{\circ}$. Third, we assume that the antennas are designed such that the variation in the radiation angle is relatively small over the frequency bandwidth of the pulse (i.e., $\Delta \theta / \Delta \beta \ll 1 / k_{0}$ ), so that $\theta \approx k_{x} / k_{0}$. Equation (9) may then be rewritten as

$$
\tilde{H}\left(k_{x}, z\right)=e^{-j k_{0} \sqrt{1-\theta^{2}} z} .
$$

The square root in the exponential function of Eq. (10) and the propagation constant in free space $k_{0}$ can be expressed by a Taylor series (around the modulation frequency $\omega_{0}$ )

$$
\begin{aligned}
& \sqrt{1-\theta^{2}} \approx 1-\frac{\theta^{2}}{2}, \\
& k_{0}(\omega)=\frac{\omega_{0}}{c}+\frac{\omega-\omega_{0}}{c},
\end{aligned}
$$

where $c$ is the speed of light in free space. In addition, the CRLH LWA angle $\theta$ may be linearized around the modulation frequency $\omega_{0}$,

$$
\theta=\xi\left(\omega-\omega_{0}\right),
$$

where $\xi \in R$ is the linearization constant parameter. This parameter may be computed as the frequency derivative of the angle at the transition frequency

$$
\begin{aligned}
\xi & =\left.\frac{\partial \theta(\omega)}{\partial \omega}\right|_{\omega=\omega_{0}} \\
& =\left.c \frac{\partial[\beta(\omega) / \omega]}{\partial \omega}\right|_{\omega=\omega_{0}}=\frac{c}{\omega_{0}}\left[\frac{1}{v_{g}\left(\omega_{0}\right)}-\frac{1}{v_{p}\left(\omega_{0}\right)}\right] \\
& =\frac{c}{\omega_{0}} \frac{1}{v_{g}\left(\omega_{0}\right)},
\end{aligned}
$$

where $v_{g}\left(\omega_{0}\right)$ is the group velocity at $\omega_{0}$ and $v_{p}\left(\omega_{0}\right)$ is the phase velocity at $\omega_{0}\left[v_{p}\left(\omega_{0}\right)=\infty\right.$ according to Eq. (4)]. ${ }^{8}$ From the definition of the group velocity, we have

$$
v_{g}\left(\omega_{0}\right)=\left[\frac{\partial \beta\left(\omega_{0}\right)}{\partial \omega_{0}}\right]^{-1}=\lim _{\omega \rightarrow \omega_{0}}\left[\frac{\beta(\omega)-\beta\left(\omega_{0}\right)}{\omega-\omega_{0}}\right]^{-1},
$$

which, using the narrow angle range assumption made above (and $\beta=k_{x}$ ), leads to

$$
v_{g}\left(\omega_{0}\right)\left(k_{x}-k_{x_{0}}\right) \approx\left(\omega-\omega_{0}\right) \text {, }
$$

where $k_{x_{0}}=k_{x}\left(\omega_{0}\right)$. Inserting this relation into Eq. (13) and then using Eq. (14) yields

$$
\theta=\xi v_{g}\left(\omega_{0}\right)\left(k_{x}-k_{x 0}\right)=\frac{c}{\omega_{0}}\left(k_{x}-k_{x_{0}}\right) .
$$

It is important to emphasize the actual conditions under which the paraxial approximation $\theta \ll 1$ (or $\Delta \theta / \Delta \beta \ll 1 / k_{0}$ ) 
used in Eq. (10) holds. Each temporal frequency component $\omega_{x}$ of the input pulse diffracts a discrete (due to periodicity) set of spatial frequencies (or plane waves) under the directions $\theta_{x, n}$ with amplitudes proportional to the Fourier coefficients (related to each antenna) of the input spatial distribution. Denoting the diffraction pattern of each $\omega_{x}$ component $\theta=\theta_{x}+\Delta \theta_{x}$, where $\Delta \theta_{x}$ is the diffraction beam width constituted by the $\theta_{x, n}$ spatial frequencies, the condition $\theta \ll 1$ requires that the bandwidth of the pulse is narrow enough or the directivity of the antennas is large enough so that $\theta_{\max }$ $+\Delta \theta_{\max } / 2 \ll 1$.

Inserting Eqs. (11), (12), and (17) into Eq. (10) finally yields the simplified transfer function

$$
\tilde{H}\left(k_{x}, z\right) \approx e^{-j\left(\omega_{0} / c\right) z} e^{-j\left[v_{g}\left(\omega_{0}\right) / c\right]\left(k_{x}-k_{x_{0}}\right) z} e^{j\left(c / 2 \omega_{0}\right)\left(k_{x}-k_{x_{0}}\right)^{2} z},
$$

where the $\left(k_{x}-k_{x 0}\right)^{3}$ term has been dropped to the first-order approximation. This equation may be rewritten as

$$
\tilde{H}\left(k_{x}, z\right) \approx e^{-j \eta_{0} z} e^{-j \eta_{1}\left(k_{x}-k_{x_{0}}\right) z} e^{j \eta_{2} / 2\left(k_{x}-k_{x_{0}}\right)^{2} z},
$$

where $\eta_{0}=\omega_{0} / c, \eta_{1}=v_{g}\left(\omega_{0}\right) / c$, and $\eta_{2}=c / \omega_{0}$ are the equivalent Taylor expansion coefficients around the modulation frequency corresponding to $\beta_{0}, \beta_{1}$, and $\beta_{2}$ in case of a dispersive medium.

To simplify the derivation of the Talbot distance, the following considerations are made. ${ }^{7}$ The first exponential in Eq. (18) may be dropped, since it is related to the modulation frequency of the pulse and does not carry any information about the envelope. The second exponential in Eq. (18) may also be dropped, since it represents a $k_{x}$-linear phase factor, equivalent in the spatial-temporal domain to the retarded frame in the time domain.

With the above simplifications and considerations, the transfer function of the system may be rewritten around $k_{x_{0}}$ as

$$
\widetilde{H}\left(k_{x}^{\prime}, z\right)=\tilde{H}\left(k_{x}=k_{x_{0}}+k_{x}^{\prime}, z\right)=e^{j\left(c / 2 \omega_{0}\right) k_{x}^{\prime 2} z} .
$$

Combining Eqs. (8) and (20), the output signal radiated at the distance $z$ is expressed in the transformed domain as

$$
\begin{aligned}
\widetilde{A}_{r}\left(k_{x}^{\prime}, z\right) & =\widetilde{A}_{a}\left(k_{x}^{\prime}, z=0\right) \widetilde{H}\left(k_{x}^{\prime}, z\right) \\
& =\Delta k_{x} \sum_{p=-\infty}^{+\infty} \tilde{A}_{e}\left(p \Delta k_{x}\right) \delta\left(k_{x}^{\prime}-p \Delta k_{x}\right) \tilde{H}\left(k_{x}^{\prime}, z\right) \\
& =\Delta k_{x} \sum_{p=-\infty}^{+\infty} \tilde{A}_{e}\left(p \Delta k_{x}\right) \delta\left(k_{x}^{\prime}-p \Delta k_{x}\right) e^{j p^{2} \phi},
\end{aligned}
$$

where

$$
\phi=\frac{c}{2 \omega_{0}} \Delta k_{x}^{2} z
$$

If the condition

$$
p^{2} \phi=2 \pi q^{\prime}=2 \pi q p^{2},
$$

with $q, q^{\prime} \in \mathbb{N}$ ( $q^{\prime}$ varies with $p$ but $q$ is constant), is satisfied, the phase factor in Eq. (21) reduces to 1, so that $\tilde{A}_{r}\left(k_{x}^{\prime}, z\right) \propto \widetilde{A}_{a}\left(k_{x}^{\prime}, z=0\right)$ according to Eq. (8), i.e., the field dis- tribution at $z$ (output) is an exact replica of the field distribution at $z=0$ (input). The corresponding position $z$ is therefore, by definition, the sought for spatial-temporal integer Talbot distance, which, combining Eqs. (22) and (23) and using $\Delta k_{x}=2 \pi / \Delta x$, reads

$$
z_{T}=\frac{2 \Delta x^{2} q}{\lambda_{0}}
$$

where $\lambda_{0}=2 \pi c / \omega_{0}$. The inverted $[\Delta x / 2$-shifted Talbot image, obtained by using $\pi$ instead of $2 \pi$ in Eq. (22)] and fractional spatial-temporal Talbot distances may then be straightforwardly obtained, following the mathematical procedure presented in (Ref. 7) as

$$
d_{s t}=\frac{s}{m} \frac{\Delta x^{2}}{\lambda_{0}}
$$

where $s, m \in \mathbb{N}$. Specifically, we have $s / m \in \mathbb{N}$ for the integer Talbot distance and its multiples, while $s / m$ is an irreducible rational number for fractional Talbot distances. At fractional Talbot distances, the periodic field distribution along the antenna array [Eq. (8)] is reproduced with a repetition rate of $m$ times that of the original distribution. The maximum value of $m$ depends on the spatial width of the pulse distribution along a single antenna $\Delta x_{p}$ [i.e., the width of $A_{e}(x, z=0)$ ], which is typically slightly larger than the spatial width of the input pulse due to CRLH dispersion, and on the spatial repetition frequency $\Delta x$, following the relation ${ }^{7}$

$$
m \leq \frac{\Delta x}{\Delta x_{p}} .
$$

If $m>\Delta x / \Delta x_{p}$, the imaged pulses overlap in space (spatial aliasing), preventing the capability of increase in the repetition rate of the original pattern.

It is important to note that the used narrow band assumption of the input pulse leads to the interpreting of the spatialtemporal Talbot effect as a combination of the conventional monochromatic spatial Talbot effect and the impulse nature of the signal, leading to Talbot zones with time-varying patterns as opposed to Talbot planes with time-invariant patterns. It should also be noted that since the energy of the pulse is decreasing around its maximum occurring located at the frequency $\omega_{0}$, the Talbot zones exhibit a Gaussian-like distribution around the maximum at the corresponding centers $z_{T}$ of the Talbot zones.

The Talbot images reproduce the propagating pulse distribution along the CRLH structures as a function of time. Moreover, a given spatial distribution is imaged at different times at the different Talbot distances $d_{s t}$ (as it will appear clearly from the results presented later in this paper). The different integer and fractional Talbot distances are known from Eq. (25). However, the image formation at each Talbot distance occurs only during a limited time duration, which corresponds to the propagation time of each pulse across each antenna element. To determine the center point of this time duration, we define a reference time $t_{d}$ as the time required for the pulse to reach the imaging distance from the generator, when it is located at the center of each antenna element. Specifically, this time reads 


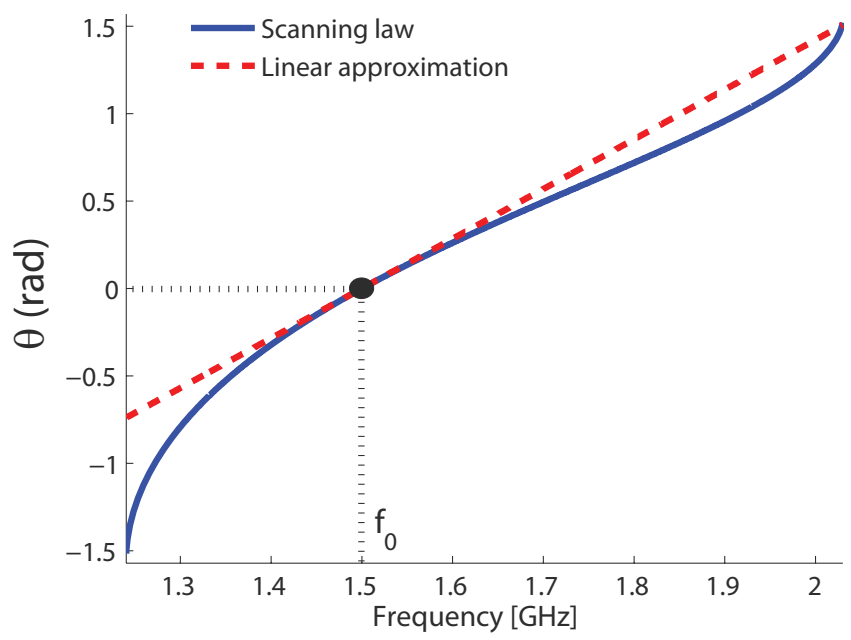

FIG. 2. (Color online) CRLH leaky-wave antenna frequency-space mapping obtained with the beam scanning relation [Eq. (3)]. This relationship is linearized around the transition frequency $\left(f_{0}=1.5 \mathrm{GHz}\right)$, using Eqs. (13) and (14).

$$
t_{d}=t_{0}+\frac{\mathrm{FWHM}}{2}+\frac{\ell}{2 v_{g}\left(\omega_{0}\right)}+\frac{d_{s t}}{c},
$$

where $t_{0}$ is the generator switch-on time, FWHM is the full width at half maximum of the pulse (at $t_{0}+\mathrm{FWHM} / 2$, the maximum of the pulse is at the input of the element), $\ell$ is the CRLH antenna element length, $v_{g}\left(\omega_{0}\right)$ is the group velocity at the modulation frequency $\left(\right.$ at $t_{0}+\mathrm{FWHM} / 2+\ell /\left[2 v_{g}\left(\omega_{0}\right)\right]$ the pulse is at the center of the element), $c$ is the speed of light, and $d_{s t}$ is the integer or fractional Talbot distance where imaging is considered.

\section{NUMERICAL RESULTS}

The results presented in this section, to demonstrate the CRLH LWA spatial-temporal Talbot phenomenon, are obtained by an exact time-domain Green's function approach. ${ }^{12,17}$ The CRLH LWA considered is composed of $N=16$ unit cells of length $p=1.50 \mathrm{~cm}(\ell=N p)$, with the circuital parameters $C_{R}=4.5 \mathrm{pF}, C_{L}=2.5 \mathrm{pf}, L_{R}=4.5 \mathrm{nH}$, and $L_{L}=2.5 \mathrm{nH}$, corresponding to a transition frequency of $f_{0}$ $=1.50 \mathrm{GHz}^{8}$ The antenna is excited by an $f_{0}$-modulated Gaussian pulse with a FWHM of 1.178 ns.

For this particular antenna, the scanning angle versus frequency relation is plotted in Fig. 2. Following Eq. (13), this curve is linearized at the transition frequency $\left(f=f_{0}\right)$. The linearization parameter is found with Eq. (14) to be $\xi$ $=0.453 \mathrm{~ns}$.

The spatial-temporal Talbot distance with antenna element spacing of $b=0.5 \mathrm{~m}$, following this linearization, is computed by Eq. (25) as $z_{T}=2.738 \mathrm{~m}$, for an infinite array. In order to validate the proposed analytical approach, Fig. 3 presents the magnitude of the field radiated by an array of 20 LWAs at the $z_{T}, z_{T} / 2$, and $z_{T} / 3$ distances as a function of the radiation direction (z-axis) of the position of the LWAs $(x$-axis) and of time. For the sake of clarity, only the region of the ten central antennas is shown. As expected, complete reconstruction of the input spatial periodic distribution is obtained at the integer Talbot distance $z_{T}$, and this same distri-

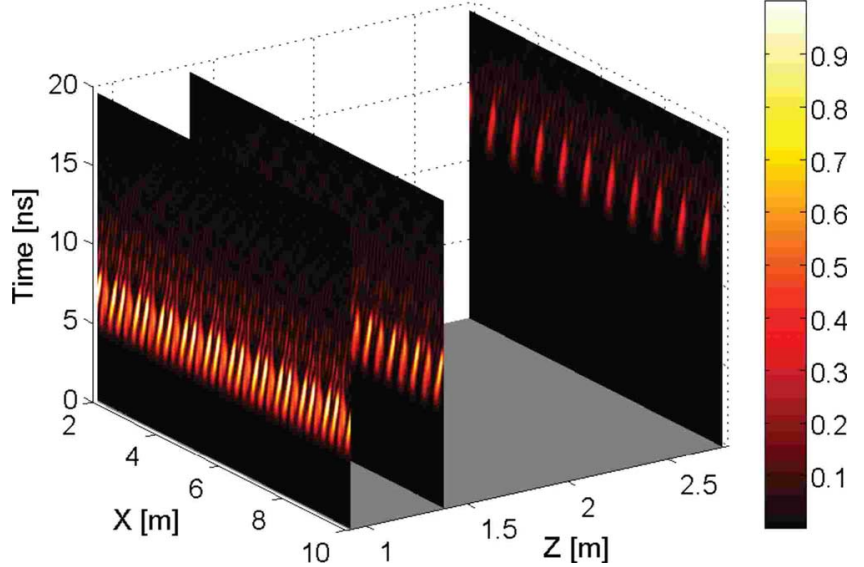

FIG. 3. (Color online) Field (magnitude) radiated by the CRLH LWA array shown in Fig. 1 (placed at $z=0$ along the $x$-axis), for antenna element spacing of $b=0.5 \mathrm{~m}$ and modulated Gaussian pulse excitation as a function of the position $x$ and time at the propagation distances $z=z_{T}=2.738 \mathrm{~m}, z$ $=z_{T} / 2=1.369 \mathrm{~m}$, and $z=z_{T} / 3=0.9127 \mathrm{~m}$.

bution with a multiplication rate of 2 and 3 is completely reconstructed at the fractional Talbot distances $z_{t} / 2$ and $z_{t} / 3$, respectively.

In order to assess the effect of array truncation, Fig. 4 presents the field radiated by an array of seven antenna elements as a function of position ( $x$-axis) and time, at a given distance $z$ from the array. Figure 4(a) shows the field magnitude obtained at the Talbot distance, where the expected seven pulses are recovered from the initial spatial pulse distribution. This reconstruction is imperfect. First, distortions occur near the edges of the array, because less frequency components contribute to imaging at the edges. Second, in contrast with the assumption made in Eq. (18), the CRLH structure is not perfectly first-order in nature, and therefore includes spurious higher-order dispersive terms that alter the image. Figure 4(b) presents the radiated field at the fractional distance $z_{T} / 2$. In this case, a double number of pulses (14) appears, validating the pulse multiplication phenomenon discussed above. Finally, Fig. 4(c) shows the radiated field at the fractional distance $z_{T} / 3$, with the expected threefold multiplication effect.

Figure 5 shows that when the number of antenna elements in the array is increased to 20 , the quality of the image reconstruction is enhanced. This is because more antennas provide more frequency contributions to each spatial point. However, small distortion effects, due to the truncation of the array (especially at the array edges) and to the high-order dispersive modes, are still visible. Figure 5(a), zoomed at the same observation region ( $x$-axis) as in the previous example, presents the radiated field at the Talbot distance, while Figs. 5(b) and 5(c) present the field at the fractionary Talbot distances $z_{T} / 2$ and $z_{T} / 3$, respectively. In all cases, the 20antenna array achieves superior image reconstruction/ multiplication as compared to the 7-antenna array.

The Talbot patterns observed in Figs. 4(a) and 5(a) are slightly titled in the $x$-time plane, with a negative slope. This effect is due neither to a numerical artifact nor to the influence of higher-order dispersion. It is due to the propagation of the pulses along the antenna elements: the energy contrib- 

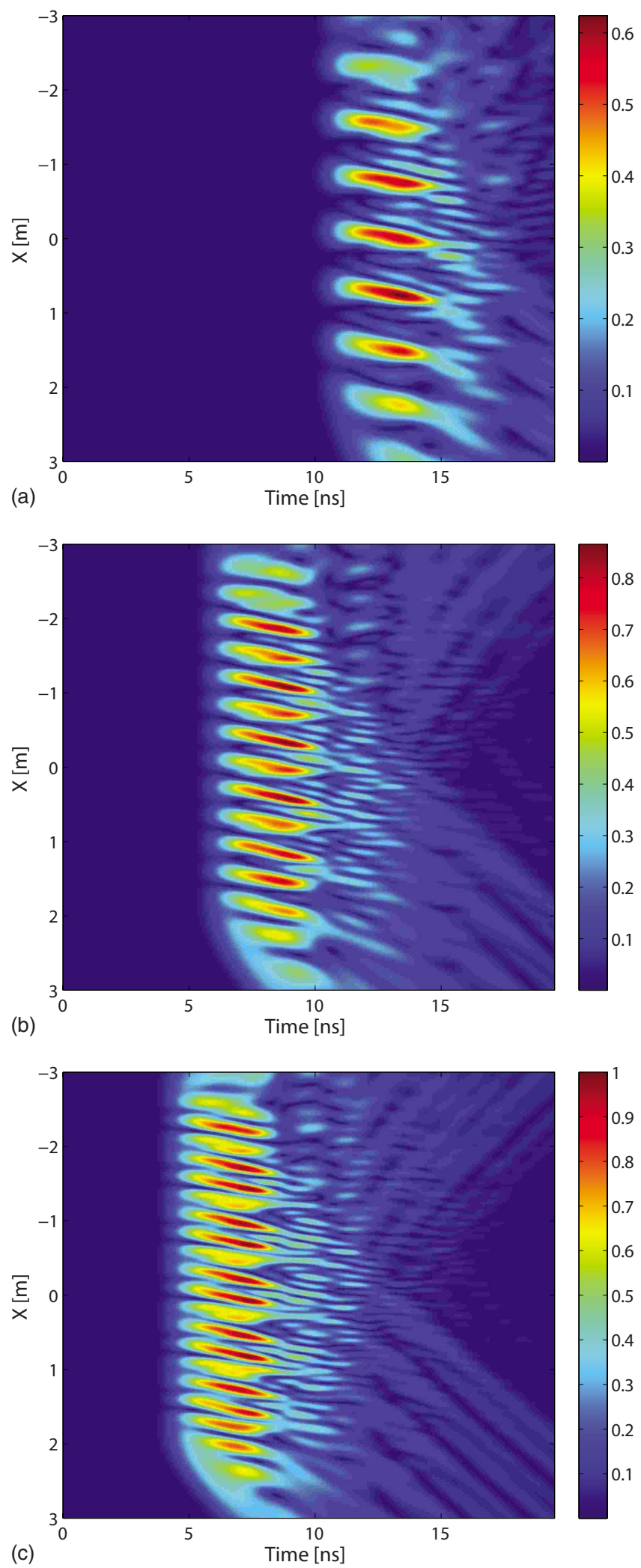

FIG. 4. (Color online) Field (magnitude) radiated by a CRLH LWA array composed of seven antenna elements (placed at $z=0$, centered at $x=0$, and fed by a modulated Gaussian pulse) at different propagation distances (z-axis). (a) $z=z_{T}=2.738 \mathrm{~m}$. (b) $z=z_{T} / 2=1.369 \mathrm{~m}$. (c) $z=z_{T} / 3=0.9127 \mathrm{~m}$.

uted by the part of the antenna elements closer to the generator is radiated earlier than the energy contributed by the part far from the generator, and therefore reaches the Talbot distance earlier. As it may be observed in Figs. 4(a) and 5(a),
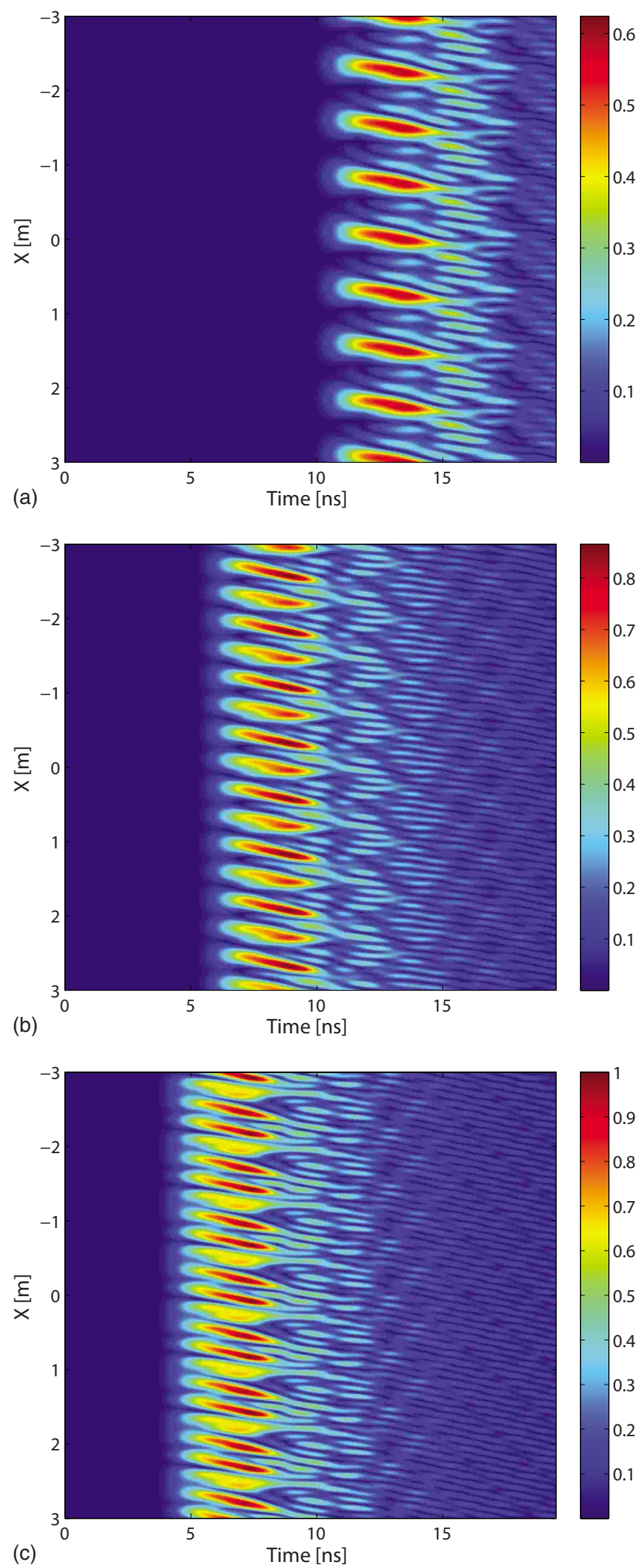

FIG. 5. (Color online) Field (magnitude) radiated by a CRLH LWA array composed of 20 antenna elements (placed at $z=0$, centered at $x=0$, and fed by a modulated Gaussian pulse) at different propagation distances ( $z$-axis). (a) $z=z_{T}=2.738 \mathrm{~m}$. (b) $z=z_{T} / 2=1.369 \mathrm{~m}$. (c) $z=z_{T} / 3=0.9127 \mathrm{~m}$.

this tilting effect becomes more and more pronounced as the Talbot distances get close to the array, because this represents an increase in the ratio between the antenna element lengths and the radiation distance. 


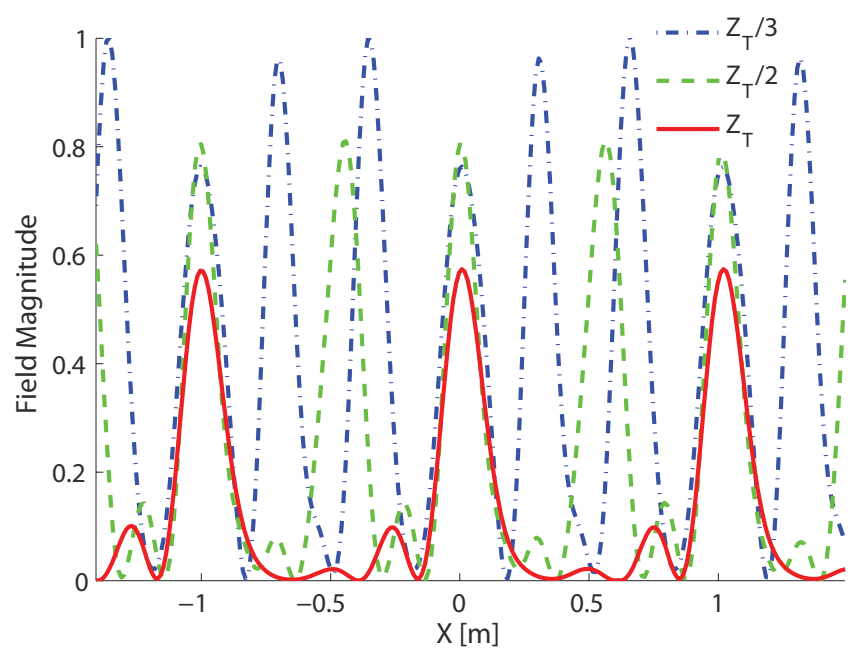

FIG. 6. (Color online) Field (magnitude) radiated by a CRLH LWA array composed of 20 antenna elements (for antenna element spacing of $b$ $=0.76 \mathrm{~m}$, placed at $z=0$, centered at $x=0$, and fed by a modulated Gaussian pulse) at the distances $z_{T}=5 \mathrm{~m}, z_{T} / 2$, and $z_{T} / 3$ computed at their reference time [Eq. (27)].

To further characterize the CRLH spatial-temporal Talbot phenomenon, let us increase to $b=0.76 \mathrm{~m}$ the antennas element spacing in the array of Fig. 5(a). The new Talbot distance is $z_{T}=5.0 \mathrm{~m}$. Figure 6 presents the radiated field at the distances $z_{T}, z_{T} / 2$, and $z_{T} / 3$ evaluated at their associated reference times $t_{d}$ [Eq. (27)]. This graph reveals two important facts. First, the repetition rates are in perfect harmonic ratios $(1,2$, and 3$)$ and perfectly synchronized at $t_{d}$. Second, the amplitude of the Talbot pattern decreases for larger distances (from $z_{t} / 3$ to $z_{t} / 2, z_{t}$, and beyond for multiples), due to free space attenuation, like in the spatial Talbot effect, but unlike in the temporal effect.

\section{CONCLUSIONS}

A spatial-temporal Talbot phenomenon, based on metamaterial CRLH leaky-wave antennas, has been presented. This phenomenon is based on the combination of the conventional monochromatic spatial Talbot effect and the transient character of the radiated pulse, which results into transient Talbot zones. This effect has been achieved in the microwave domain using the radiation properties of CRLH LWA array. A complete analytical formulation of this Talbot phenomenon has been presented and numerically verified using an exact time-domain Green's function approach.

\section{ACKNOWLEDGMENTS}

This work was partially supported by the Spanish Ministry of Education and Science under Grant No. FPUAP2006-015. The authors wish to thank Dr. J. Azaña for fruitful discussions.

${ }^{1}$ H. F. Talbot, Philos. Mag. 9, 401 (1836).

${ }^{2}$ A. W. Lohmann, Optik (Stuttgart) 79, 41 (1987).

${ }^{3}$ L. Liu, Opt. Lett. 14, 1312 (1989).

${ }^{4}$ C. Zhou, S. Stankovic, C. Denz, and T. Tschudi, Opt. Commun. 161, 209 (1999).

${ }^{5}$ J. T. Winthrop and C. R. Worthington, J. Opt. Soc. Am. 55, 373 (1965).

${ }^{6}$ N. Guérineau, B. Harchaoui, and J. Primot, Opt. Commun. 180, 199 (2000).

${ }^{7}$ J. Azaña and M. A. Muriel, IEEE J. Sel. Top. Quantum Electron. 7, 728 (2001).

${ }^{8}$ C. Caloz and T. Itoh, Electromagnetic Metamaterials: Transmission Line Theory and Microwave Applications (Wiley, New York/IEEE, New York, 2005).

${ }^{9}$ S. Abielmona, S. Gupta, and C. Caloz, IEEE Microw. Wirel. Compon. Lett. 17, 864 (2007).

${ }^{10}$ S. Gupta and C. Caloz, in Proc. of International Symposium on Electromagnetic Theory (EMTS), Ottawa, 2007, CD-ROM. Available online at: www.ursi.org/B/EMTS_2007/O18-14/1-Gupta046.pdf

${ }^{11}$ S. Gupta, C. Caloz, and S. Abielmona, in Proc. of IEEE MTT-S International Microwave Symposium Digest, Atlanta, 2008, pp. 807-810.

${ }^{12}$ J. S. Gómez-Díaz, A. Álvarez-Melcón, and C. Caloz, in Proc. IEEE Antenna and Propagation Symposium, San Diego, 2008, pp. 1-4.

${ }^{13}$ J. Azaña and M. A. Muriel, Opt. Lett. 24, 1672 (1999).

${ }^{14}$ N. K. Berger, B. Vodonos, S. Atkins, V. Smulakovsky, A. Bekker, and B. Fischer, Opt. Commun. 217, 343 (2003).

${ }^{15}$ A. Oliner and D. R. Jackson, Antenna Engineering Handbook, 4th ed. (McGraw-Hill, New York, 2007).

${ }^{16}$ B. E. A. Saleh and M. C. Teich, Fundamentals of Photonics, 2nd ed. (Wiley, New York, 2007).

${ }^{17}$ X. H. L. Zhou and C. Chan, Photonics Nanostruct. Fundam. Appl. 3, 100 (2005). 